\title{
TrackAER: Real-Time Event-Based Particle Tracking
}

\author{
Alexander Rusch ${ }^{1 *}$, Thomas Roesgen ${ }^{1}$ \\ ${ }^{1}$ ETH Zurich, Institute of Fluid Dynamics, Zurich, Switzerland \\ *arusch@ethz.ch
}

\section{Introduction \& Related Work}

Event-based cameras (Lichtsteiner et al., 2008; Posch et al., 2010; Gallego et al., 2020) operate fundamentally different from frame-based cameras: Each pixel of the sensor array reacts asynchronously to relative brightness changes creating a sequential stream of events in address-event representation (AER). Each event is defined by a microsecond-accurate time stamp, the pixel position and a binary polarity indicating a relative increase or decrease of light intensity. Thus, event-based cameras only sense changes in a scenery while effectively suppressing static, redundant information. This renders the camera technology promising also for flow diagnostics. In established approaches like PIV or PTV vast amounts of data are generated, only for a large part of redundant information to be eliminated in data post-processing. In contrast, eventbased cameras effectively compress the data stream already at the source. To make full use of this potential, new data processing algorithms are needed since event-based cameras do not generate conventional framebased data. This work utilizes an event-based camera to identify and track flow tracers such as helium-filled soap bubbles (HFSBs) with real-time visual feedback in measurement volumes of the order of several cubic meters.

The utility of event-based cameras for flow diagnostics has been demonstrated in a few studies already. Drazen et al. (2011) used one of the first dynamic vision sensors (DVS) available for a proof-of-concept study showing that $2 \mathrm{D}$ particle tracking velocimetry is possible for pipe flows with solid particles as tracers. $\mathrm{Ni}$ et al. (2012) performed particle tracking with event-based cameras in a microscopic environment. In order to distinguish microspheres from noise, they applied a circle Hough transform. Borer (2014) and Borer et al. (2017) extended the idea of event-based particle tracking to a larger domain of interest and used multiple event-based cameras for volumetric PTV measurements. Data sets were generated in a 3D calibrated setup of three DVS cameras and processed off-line. Their tracking approach made use of a Kalman filter for track prediction and relied on triangulating particle tracks after identifying them in each camera's individual data stream. Wang et al. (2020) used an event-based stereo camera setup for volumetric PTV measurements imposing an incompressibility constraint for regularization. Their approach was evaluated using simulated data and with measurements in a centimeter-sized, water-filled cavity.

\section{Real-Time Algorithm}

There are two basic ways of processing the asynchronous event data stream: In one approach, events are accumulated in fixed time windows or fixed-size batches followed by processing steps using pseudo-frames reconstructed from the events. While this allows the application of well-established frame-based computer vision algorithms, it also implies a loss of the high temporal accuracy and low latency of event-based cameras. Alternatively, events can be processed in a fully sequential manner, i.e., "event-by-event", keeping the precise timing information and ordering of the events. No frames are reconstructed that could interface to classical computer vision algorithms for further processing. In this case, event-based algorithms are required that analyze the flow (motion) information contained in the asynchronous event stream. Our work follows such a per-event approach, preserving the speed and scalability of the technology. The key to identifying and tracking a moving object from the event stream is to identify coherent spatio-temporal event activity. Each incoming event may be added to a cluster, updating that cluster's position and the list of contributing events. If no assignment can be made, the event information is registered to prepare a trigger for the future creation of a new cluster. Stale events are pruned from existing clusters using an "age" criterion. This may happen in response to an occlusion in the scenery or when a moving object leaves the field of view. The algorithm self-supervises its performance and may selectively skip processing steps if necessary for real-time responsiveness. 


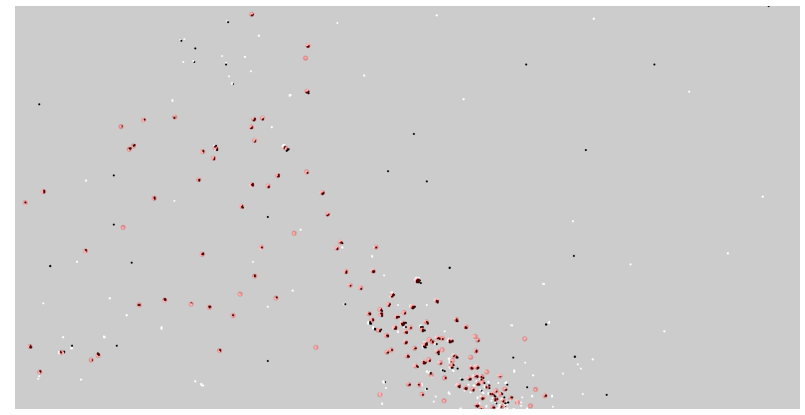

(a) TrackAER event snapshot (16 ms time window). Events are displayed indicating positive (white) and negative (black) polarity. Tracked clusters are overlaid in red.

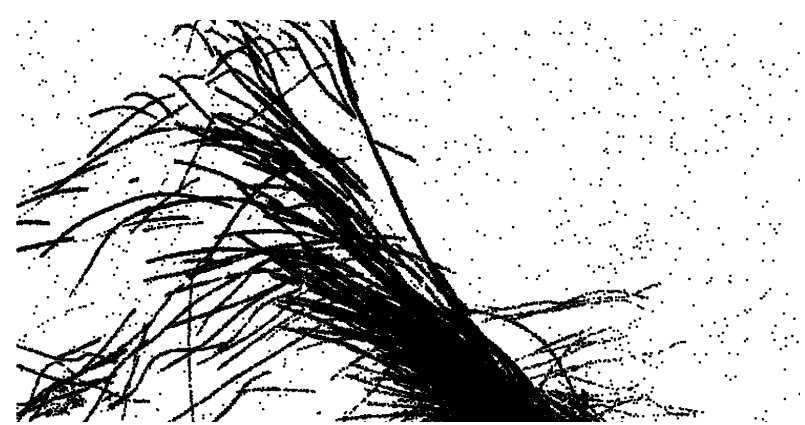

(b) Pseudo-streak integration from events over $10 \mathrm{~s}$ to visualize particle trajectories. Only every fifth event is visualized and event polarity is not indicated.

Figure 1: Visualization (field of view approx. $3.5 \mathrm{~m} \times 2.0 \mathrm{~m}$ ) of the flow caused by an indoor ventilation system. Helium-filled soap bubbles are introduced at the bottom of the scene and convected.

\section{Results \& Conclusion}

For visualization only, pseudo-frames can be generated from the event stream and fused with the movement of the cluster trackers. No frames are used at any stage of the tracking algorithm. Figure 1 shows results from a study of an indoor ventilation system. A room is partially seeded with HFSBs that move due to the flow induced by the ventilation system. Events shown in the background are measurement noise. TrackAER successfully ignores these spurious events and does not interpret them as tracer particles. More than 100 HFSBs are successfully tracked simultaneously and in real-time.

Our new real-time event-based detection and tracking algorithm, TrackAER, provides immediate insight into flow fields during measurements. Tracer particles are successfully detected and tracked in volumes of several cubic meters, whereas noise from the camera sensor and the scenery are effectively eliminated. Ongoing research focuses on the actual flow field reconstruction as well as on the extension of the system to volumetric measurements.

\section{References}

Borer D (2014) 4D Flow Visualization with Dynamic Vision Sensors. Ph.D. thesis. ETH Zurich

Borer D, Delbruck T, and Rösgen T (2017) Three-dimensional particle tracking velocimetry using dynamic vision sensors. Experiments in Fluids 58:165

Drazen D, Lichtsteiner P, Häfliger P, Delbrück T, and Jensen A (2011) Toward real-time particle tracking using an event-based dynamic vision sensor. Experiments in Fluids 51:1465-1469

Gallego G, Delbruck T, Orchard GM, Bartolozzi C, Taba B, Censi A, Leutenegger S, Davison A, Conradt J, Daniilidis K, and Scaramuzza D (2020) Event-based vision: A survey. IEEE Transactions on Pattern Analysis and Machine Intelligence

Lichtsteiner P, Posch C, and Delbruck T (2008) A $128 \times 128120 \mathrm{db} 15 \mu$ s latency asynchronous temporal contrast vision sensor. IEEE Journal of Solid-State Circuits 43:566-576

Ni Z, Pacoret C, Benosman R, Ieng S, and Régnier S (2012) Asynchronous event-based high speed vision for microparticle tracking. Journal of Microscopy 245:236-244

Posch C, Matolin D, and Wohlgenannt R (2010) A qvga 143 db dynamic range frame-free pwm image sensor with lossless pixel-level video compression and time-domain cds. IEEE Journal of Solid-State Circuits 46:259-275

Wang Y, Idoughi R, and Heidrich W (2020) Stereo event-based particle tracking velocimetry for 3d fluid flow reconstruction. in European Conference on Computer Vision. pages 36-53. Springer 